between Trx and MIF in adults with SIRS/sepsis. (Leaver 2009) Furthermore Trx was shown to inhibit the secretion of MIF in THP1 cells (Tamaki 2006). The aim of this study was to determine the effect of exogenous Trx on the release of MIF and for comparison IL8 and IL-10 from primary human monocytes at baseline and following stimulation with lipoteichoic acid (LTA) or lipopolysaccharide (LPS).

Methods Monocytes were extracted from whole blood of healthy volunteers using Percoll gradients and MACS columns. Monocytes $\left(1 \times 10^{6}\right.$ cells $\left./ \mathrm{ml}\right)$ were pre-incubated with Trx $(0.1-10000 \mathrm{nM})$ for $24 \mathrm{~h}$ followed by treatment $(24 \mathrm{~h}$ ) with medium alone, LPS $1 \mu \mathrm{g} / \mathrm{ml}$, LTA $10 \mu \mathrm{g} / \mathrm{ml}$. MIF, IL-8 and IL-10 concentrations in cell supernatants were determined by ELISA.

Results Following incubation with Trx there was no significant change in MIF release from monocytes. By contrast, LPS and LTA significantly $(p<0.01)$ induced MIF from base line. When monocytes were treated with LPS (Abstract S51 Figure 1) or LTA following preincubation with Trx, MIF release was significantly less than the theoretical additive effects of the two treatments alone. By contrast, although Trx significantly induced IL-8 and IL-10, Trx did not modulate LPS or LTA induced cytokine release.

Conclusion Trx reduced MIF release following stimulation with LPS and LTA. Extracellular Trx exerts an anti-inflammatory effect in this model. The Trx/MIF axis should be explored as a potential route for therapeutic intervention in patients with sepsis.

\section{S52 THE ROLE OF THE RECEPTOR FOR ADVANCED END PRODUCTS (RAGE) IN ACUTE LUNG INJURY (ALI)}

doi:10.1136/thx.2010.150938.3

${ }^{1} \mathrm{~B}$ A Thompson, ${ }^{2} \mathrm{C}$ W Stitt, ${ }^{1} \mathrm{D}$ F McAuley, ${ }^{1} \mathrm{C}$ M O'Kane. ${ }^{1}$ Centre for Infection and Immunity, Queen's University Belfast, Belfast, UK; ${ }^{2}$ Centre for Vision and Vascular Sciences, Queens University Belfast, Belfast, UK

Introduction RAGE is expressed by both alveolar epithelial and endothelial cells. The receptor can bind to pro-inflammatory ligands, including HMGB1 which is elevated in ALI. RAGE negative mice show a reduced inflammatory response when challenged with LPS. We hypothesised that RAGE activation in the pulmonary circulation propagates inflammation in ALI and that soluble RAGE (sRAGE) can act as a scavenger receptor, reducing RAGE-mediated inflammation.

Aim To investigate HMGB1 RAGE-dependent activation of pulmonary microvascular endothelial cells, and resultant production of inflammatory cytokines and tissue-destructive proteases in a model of ALI.

Methods Primary HPMECs (human pulmonary microvascular endothelial cells) were stimulated with clinically relevant concentrations of HMGB1. MAPK phosphorylation was assessed by Western Blot. Supernatants collected at $72 \mathrm{~h}$ were analysed by ELISA for IL-8, Tissue Inhibitors of Metalloproteinases (TIMPs)-1 and -2 . Experiments were repeated both with a RAGE-blocking antibody and in the presence of sRAGE.

Results HMGB1 increased phosphorylation of ERK1/2 at $15 \mathrm{~min}$ and p38 at 30 min after stimulation HMGB1 increased IL- 8 secretion (from $6.46 \mathrm{ng} / \mathrm{ml}$ to $8.75 \mathrm{ng} / \mathrm{ml}, \mathrm{p}<0.01$ ) and significant decrease in TIMP-1 secretion (from $19.79 \mathrm{ng} / \mathrm{ml}$ to $16.9 \mathrm{ng} / \mathrm{ml}, \mathrm{p}<0.05$ ) at $72 \mathrm{~h}$. MAPK activation, IL-8 increase and TIMP-1 decrease was significantly reversed in the presence of sRAGE $(p<0.05)$ (Abstract S52 Figure 1). Incubating cells with a RAGE blocking antibody inhibited MAPK phosphorylation by HMGB1.

Conclusions Data suggest that RAGE ligation leads to an increase in pulmonary endothelial cell activation and IL-8 release. In vivo this would increase inflammatory cell influx into the pulmonary environment, propagating inflammation. This is combined with a decrease in TIMP protection potentially increasing degradation of the basement membrane by functionally unopposed proteinases.

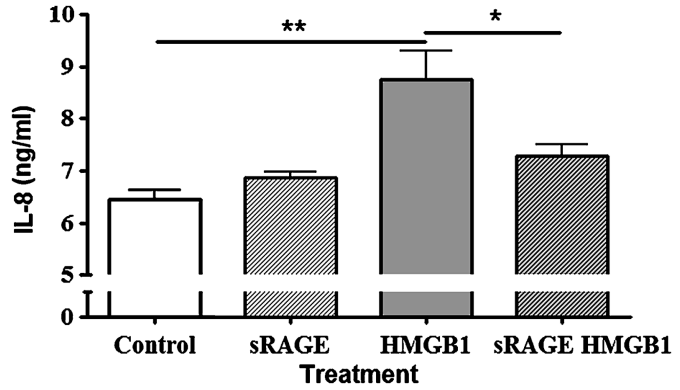

Abstract S52 Figure 1 sRAGE and HMGB1 were used at $1 \mu \mathrm{g} / \mathrm{ml}$. Statistics are one way ANOVA with Tukey post test. ${ }^{*} \mathrm{p}<0.05$, ${ }^{* *} \mathrm{p}<0.01$.

That these changes can be partially rescued using sRAGE shows that it could potentially decrease inflammatory damage in ALI.

\section{S53 NANOPARTICLES CAUSE PULMONARY INFLAMMATION THROUGH IL-1 $\alpha$ AND PARTIAL ACTIVATION OF THE NLRP3 INFLAMMASOME}

doi:10.1136/thx.2010.150938.4

${ }^{1} \mathrm{~N}$ R Riteau, ${ }^{2} \mathrm{~A}$ Y Yazdi, 'L B Baron, 'A G Gombault, ${ }^{2} \mathrm{G}$ G Guarda, ${ }^{2} \mathrm{~J}$ T Tschopp, II C Couillin. 'Laboratory of Molecular Immunology and Embryology, University of Orleans and Centre National de la Recherche Scientifique, Orleans, France; ${ }^{2}$ Department of Biochemistry, University of Lausanne, Epalinges, Switzerland

Nanoparticles are increasingly used in various fields, including biomedicine and electronics. Their size and physical characteristics allow them to easily access the cytosol of tissue or immune cells. Although inorganic metal oxide nano- $\mathrm{TiO}_{2}$ is believed to be biologically inert, an emerging literature reports increased incidence of respiratory diseases in exposed people. Here, we show that instillation of nano- $\mathrm{TiO}_{2}$ induces lung inflammation which is strongly suppressed in IL-1R- and IL- $1 \alpha$-deficient mice. They have drastically reduced neutrophil recruitments in the alveolar space, together with lung inflammatory cytokine productions. Surprisingly, the NLRP3 inflammasome complex seems to be only partially involved. Nlrp3-, ASC- or Casp-1-deficient mice show only a slight reduction in pulmonary inflammatory response. IL-1 $\beta$-deficient mice exhibit decreased inflammation parameters that are less pronounced than IL-1 $\alpha$-deficient mice. In vitro experiments show that primary pulmonary epithelial cells cultured in presence of nano- $\mathrm{TiO}_{2}$ are able to produce KC and IL- $1 \alpha$, but not IL- $1 \beta$, to initiate inflammation. In conclusion, it appears that nanoparticles-mediated inflammation is highly dependent on IL- $1 \alpha$ and to a less extend on the NLRP3 inflammasome/IL-1 $\beta$ axis. Collectively, these data demonstrate that the expending use of nano- $\mathrm{TiO}_{2}$, e.g. in cosmetics, may present a health hazard and should be taken under consideration, a situation reminiscent of inflammation provoked by asbestos exposure.

\section{Orphan lung diseases S54 CHARACTERISING SARCOIDOSIS USING A WEB-BASED REGISTRY: A PILOT STUDY}

doi:10.1136/thx.2010.150938.5

R K Coker, C Wang, A Sepahzad, N J Roberts, M R Partridge. Imperial College London, NHLI Division at Charing Cross and Hammersmith Hospitals, London, UK

Introduction and Objectives Sarcoidosis is a chronic multisystem disorder of unknown cause. Demographic and phenotypic characteristics have not been comprehensively studied in Britain. 
Extrapolation from a North American population (ACCESS) may not be valid. This retrospective pilot study aimed to evaluate the potential of a novel web-based registry, by recording demographic and clinical characteristics, follow-up and treatment.

Methods 199 potential patients were identified from specialist respiratory clinics at two hospitals. 135 case notes were reviewed. Data were extracted from first and follow-up visits with random data quality checks by at least one other author.

Results Of 135 patients, 55\% were female and 47\% Afro-Caribbean; mean age (years) was $43.7 \pm 12.3$ and patients had on average three follow-up visits per year. Presenting symptoms included cough (95\%), breathlessness (41\%), skin rashes $(22 \%)$, arthralgia $(15 \%)$, fatigue (19\%), eye symptoms (14\%) and fever (11\%). $14 \%$ had stage 0 chest radiographs, $46 \%$ stage I, $16 \%$ stage II, $15 \%$ stage III and $9 \%$ stage IV. $45 \%$ had raised serum angiotensin-converting enzyme at first visit. $80 \%$ had histological confirmation. Systemic corticosteroids were the commonest treatment (21.5\% after first visit). Cutaneous involvement was highest in females (64\%) and AfroCaribbeans (48\%). Nodular lesions were the most common; $77 \%$ had granulomas on skin biopsy. $94 \%$ of those with skin disease had other organ involvement and received on average three treatments (topical $77 \%$, prednisolone $64 \%$, and hydroxychloroquine $45 \%$ ). During follow-up, 73\% reported improvement. 20 patients had definite/ probable neurosarcoid (Zajicek criteria). Symptoms included parasthesiae $(45 \%)$, visual disturbance $(30 \%)$, headache $(30 \%)$, seizures $(10 \%)$ and anosmia $(5 \%)$. Four had a VIIth nerve palsy. Diagnosis was made on MRI in $95 \% ; 20 \%$ had CNS biopsies. $80 \%$ received prednisolone and $25 \%$ intravenous methylprednisolone. $15 \%$ were discharged after remission. Of the rest, $40 \%$ deteriorated during follow-up, $40 \%$ stabilised and $20 \%$ improved. Raised serum ACE was common in those with cutaneous and neurological disease.

Conclusions A novel web-based registry is a valuable method of recording clinical data in sarcoidosis. Additional use by other centres and the prospective recording of details of new cases could provide useful epidemiological data for the UK and drive future hypothesisbased studies.

\section{S55 PULMONARY FIBROSIS WITH AND WITHOUT CO-EXISTING CONNECTIVE TISSUE DISEASE: DOES IT AFFECT SURVIVAL?}

doi:10.1136/thx.2010.150938.6

${ }^{1} \mathrm{~V}$ Navaratnam, ${ }^{2} \mathrm{~N}$ Ali, ${ }^{1} \mathrm{CJP}$ Smith, ${ }^{1} \mathrm{~A}$ Fogarty, ${ }^{1} \mathrm{R}$ B Hubbard. ${ }^{1}$ Divisional of Epidemiology, University of Nottingham, Nottingham, UK; ${ }^{2}$ Queens Medical Centre, Nottingham University Hospitals Trust, Nottingham, UK

Introduction The impact of co-existing connective tissue disease on the survival of patients with pulmonary fibrosis remains unclear, since studies into the subject have so far yielded varying results. This study compares the survival of individuals with idiopathic pulmonary fibrosis (IPF) to those with connective tissue disease associated pulmonary fibrosis (CTD-PF) using data from The Health Improvement Network (THIN), a large general practice database in the UK.

Methods Incident cases of individuals with IPF and CTD-PF between the years 2000 and 2008 were identified. Survival analysis was performed on this dataset using Kaplan-Meier methods and Cox regression was used to compare mortality rates between patients with IPF and CTD-PF, adjusting for age and gender.

Results During the study period, there were 2208 incident cases of IPF, who were predominantly male $(64 \%)$ and had a mean age at time of diagnosis of 73.9 years (95\% CI 73.5 to 74.4 years). In comparison there were 318 incident cases of CTD-PF, of which the majority were female $(56 \%)$ and the mean age at time of diagnosis was 67.8 years (95\% CI 66.6 to 68.9). The commonest connective tissue disease associated with pulmonary fibrosis was rheumatoid arthritis (211 cases), followed by systemic sclerosis (39 cases). The

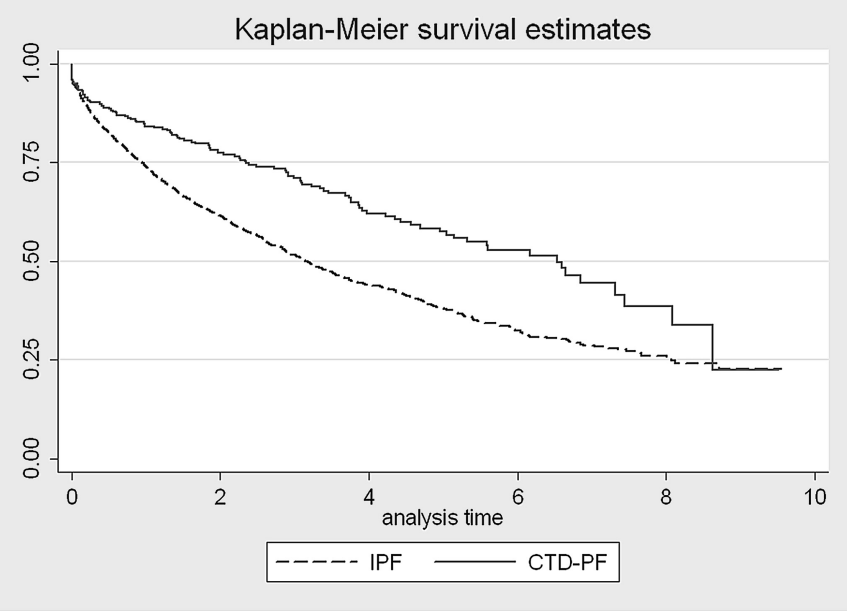

Abstract S55 Figure 1 Kaplan-Meier survival estimates.

mean follow-up period after diagnosis for our cohort was 2.4 person years and during this period 1117 (51\%) patients with IPF and 116 (36\%) patients with CTD-PF had died. The mortality rates for patients with IPF and CTD-PF were 221 (95\% CI 208 to 234) and 121 (95\% CI 101 to 146) deaths per 1000 person years respectively. The median survival for patients with CTD-PF was significantly higher at 6.5 years compared to 3.2 years in patients with IPF $(p=0.0033)$ (See Abstract S55 Figure 1). After adjusting for age and gender, patients with IPF had a worse prognosis compared to those with CTD-PF (HR 1.32, 95\% CI 1.08 to 1.60 ).

Conclusion Individuals who have CTD-PF have a better prognosis after diagnosis when compared to those with IPF. This may relate to the differing natural histories of the diseases and needs further investigation.

\section{S56 FLAT TRACHEA SYNDROME: AN UNDER-DIAGNOSED AND UNDER-TREATED CONDITION?}

doi:10.1136/thx.2010.150938.7

G Niranjan, J F K Marzouk. University Hospital of Coventry and Warwickshire, Coventry, UK

Objectives Flat Trachea Syndrome (Tracheobronchomalacia) is a central airway disease characterised by weakness of the wall and dynamic decrease in the tracheal lumen and the large bronchi, particularly while exhaling. It causes chronic symptoms such as cough, dyspnoea, increase in recurrent infections, and poor secretion management, but it can also progress to chronic respiratory failure and death. It remains largely under-diagnosed unless clinicians are thoroughly acquainted with its peculiar symptomology being usually confused with other common diseases like chronic obstructive pulmonary disease (COPD) or asthma. Surgery with posterior tracheobronchial splinting (tracheobronchoplasty) using a polytetrafluoroethylene (PTFE) Teflon patch has been considered as a possible treatment option for this condition.

Methods A retrospective series of 28 patients that underwent tracheobronchoplasty using a PTFE Teflon patch between July 1998 and July 2010 were evaluated for symptoms, lung capacity and exercise capacity.

Results The age range of patients treated was from 24 to 82 (mean 66). $61 \%$ (17) of those treated were men, with all cases being diagnosed with obstructive airways disease on spirometry, with the FEV1 ranging from $34 \%$ to $65 \%$ pre-operatively. 25 patients (89\%) presented with severe dyspnoea, 23 patients $(82 \%)$ with uncontrollable cough, and 21 patients $(75 \%)$ reported recurrent pulmonary infections. There was no in-hospital or 30-day mortality. After surgery symptomatic 